\title{
OPTIMAL CONTROL OF THE TOTAL ARTIFICIAL HEART USING HALBACH ARRAY LINEAR MOTOR
}

\author{
A. E. ElGebaly, M. El-Nemr, and E. M. Rashad \\ Faculty of Engineering, Tanta University, Tanta, EGYPT \\ E-Mail:enggebaly@hotmail.com,nemrit@yahoo.com
}

\begin{abstract}
The total artificial heart (TAH) is a mechanical device to be integrated with mammal's blood flow dynamics to reinstate the functionality of biologically defected heart. In this paper, a Halbach array linear synchronous motor (HALSM) is used as a TAH. In this paper, the optimal control of the TAH that utilizes HALSM is studied to investigate its performance driving blood circulation. The optimal control system is relied on enabling the TAH of following certain pattern of blood flow. Analytical formulas for the HALSM developed force is presented depending on space harmonic analysis for the solution of the multilayer boundary field problem. Parameters of the formulas are obtained by means of magnetic field analysis using finite element method (FEM). The dynamic model of the blood circulation driven by HALSM is developed as a main step before control system design. PID controller is designed to control the TAH system. The genetic algorithm (GA) is used to obtain the optimized controller parameter .Simulated study is carried out for the TAH optimal control as integrated element in the blood cycle of an adult human. The obtained results, presents valuable guide for the design of TAH control system implementing Halbach linear motors.
\end{abstract}

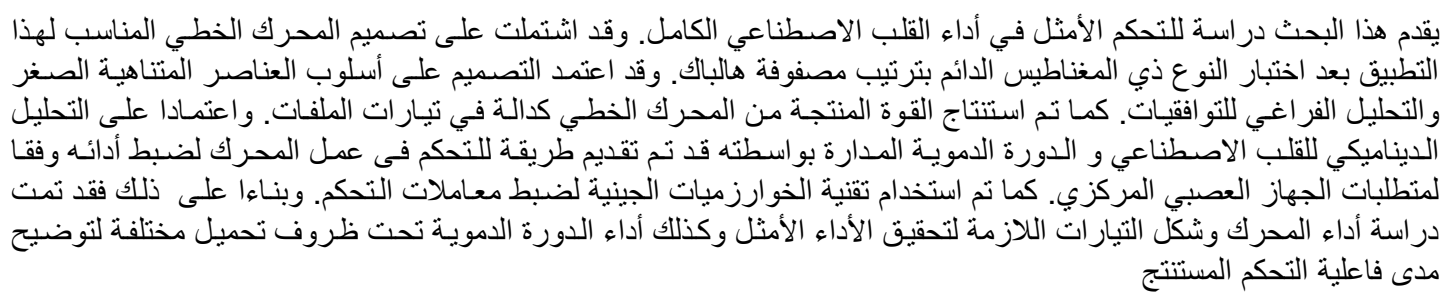

Keywords: Total Artificial Heart, Halbach array linear motor, Finite element method, Genetic Algorithm, blood circulation dynamics

\section{Introduction}

The total artificial heart (TAH) is one of the most vital actuators for the life of some heart patients. The TAH is a mechanical device to be integrated with blood flow dynamics to restore the functionality of biologically defected heart. Such utility is critical to maintain patient's life until heart transplantation. The design and control of this actuator present a big challenge for researchers in engineering and medical science [1].

The design of such actuator should fulfill the volume and weight limitation with minimum energy loss and highest possible efficiency [2]. Moreover, it should be able to produce sufficient force to keep the blood circulation in correspondence to the nervous system commands [3]. Moreover, it should be fault tolerated to allow avoiding total breakdowns of the system, which is literally a matter of life or death. Typically, TAH should be furnished with robust controller that is integrated with nervous system and the blood circulation parameters.

Conventional TAH systems implement rotary brushless DC motors and a conversion gear mechanism, contains many moving components to drive the blood circulation. These TAHs produce linear motion to compress blood through its circulation. These types have been associated with the gear mechanism problems such as reliability and large volume [2].

Linear motor actuator does not require bearings or a system to convert the motion. So, more reliability and less design complication will be provided. Reliability is even improved by using a linear motor actuator with no 
movable current-currying coils. Thus, the device can be used for momentary and permanent therapy [4].

Halbach array [5] is used to develop a TAH linear motor actuator because this concept fulfills some of basic requirements. This TAH depend on Halbach array linear motor configuration due to its advantages of having static coil and moving permanent magnets are used. Halbach array concept does not require back iron or have cogging force.

Fig. 1 illustrates the different parts of a TAH with a HALSM. Analog to the natural heart, TAH comprise two blood chambers. To pump the blood into the aorta or pulmonary artery, pusher plates compress these blood chambers. Additional component can also be added such as one directional valve in the beginning and end of two chambers.

TAHs are implanted into the thorax in place of the weak natural heart. The available space is therefore limited. Characteristics of this Halbach array actuator was roughly studied by Thomas Finocchiar et al [2]. Finite-element method (FEM) has been used to obtain suitable dimensions and characteristics [4]. Design has taken into consideration obtaining required force at minimum amount of losses. Also, coils excitation has been obtained along full TAH beat.

In this paper, HALSM developed force is formulated in equation to involve the motor in the dynamic model of overall system. The dynamic model of blood circulation system driven by HALSM is established as a first step to controller design. The GA is utilized as a second step to obtain robust control parameters using proper performance index. The performance of the TAH is illustrated under these optimized parameters.

\section{2- HALSM for Artificial Heart System}

As shown in Fig.1, the TAH compresses blood into two paths the first is the aorta and the second is the pulmonary

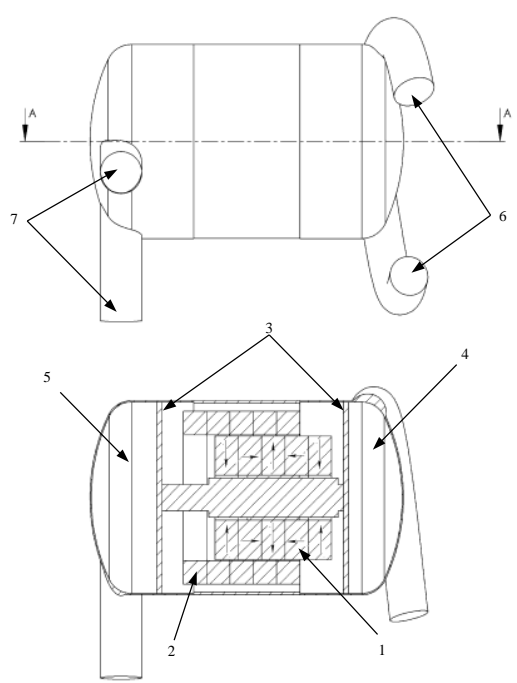

Fig.1: Components of TAH with HALSM: 1 PM, 2 coils, 3 pusher plates, 4 aorta (left) chamber, 5 pulmonary (right) chamber, 6 left chamber terminals, 7 right chamber terminals artery. Each of two phases has its own hemodynamics parameters [6]. Hemodynamics is the branch of physiology that studies the circulation of the blood and the forces involved. Each phase requires special force to pumping blood. The average blood flow rate for an adult human equal $75 \mathrm{ml}$ per beat. The blood flow rate takes the

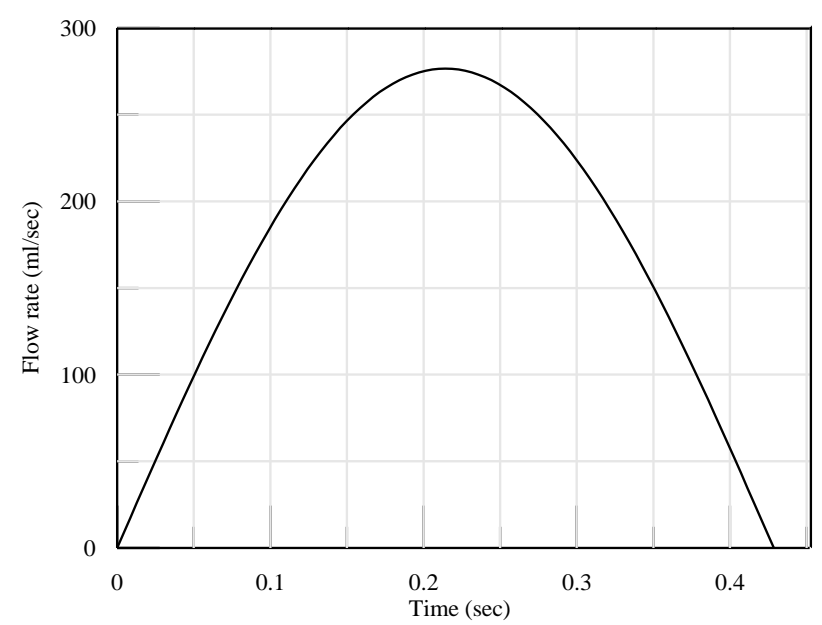

Fig.2: Demand blood Flow rate

form of sinusoidal wave as shown in Fig. 2 where the TAH provide 70 beat per minute (bpm). The blood flow rate in each of two heart chambers is the same. According to Starling's law, the volume of each two chambers is the same [6].

The HALSM is an ironless drive where the magnets are aligned in a special manner known as Halbach array. Through this alignment, the magnetic flux density is augmented on the outer side, while it is partially cancelled on the inner side of the magnets. NdFeB52 permanent magnet is used in this model because it has good characteristics, coercivity of $891300 \mathrm{~A} / \mathrm{m}$, for obtaining high magnetic field for small model. Coils are arranged from left to right as coil 1 followed by coil 2, coil3, coil 4 and coil 5. Each coil of the five coils has 1500 turns of copper 30 AWG [4].

The HALSM for the TAH has the following dimensions $66 \mathrm{~mm}$ outer diameter, $16 \mathrm{~mm}$ inner diameter, and a height of $45 \mathrm{~mm}$. It has a weight of $1 \mathrm{~kg}$. So, it is suitable to be implanted into the human body [4]. Each of two blood chambers has a volume of $75 \mathrm{ml}$ and the pusher plate has an area of $0.004167 \mathrm{~m}^{2}$. The distance of each stroke is $18 \mathrm{~mm} \mathrm{[4].}$

\section{3- Electromagnetic Force Calculation}

The analytical model of Halbach array linear motor developed force depends on the analysis of MMF produced by each of PM and five coils[7]. Fig. 3 shows a longitudinal cross-section of Halbach array linear motor. It is assumed that the PM is magnetized constantly with coercive force $H_{c}$ in radial direction. $H_{c}$ can be represented in Fourier series as a function of displacement $x$ as: 


$$
H_{c}(x)=\sum_{n=1,3, . .} H_{c n} \cos \left(n \frac{\pi}{\tau} x\right)
$$

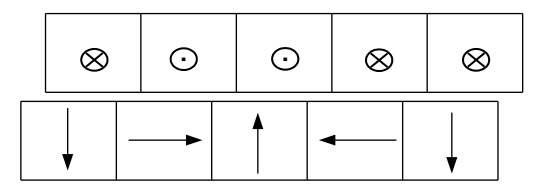

a) Longitudinal cross section of Halbach array linear motor

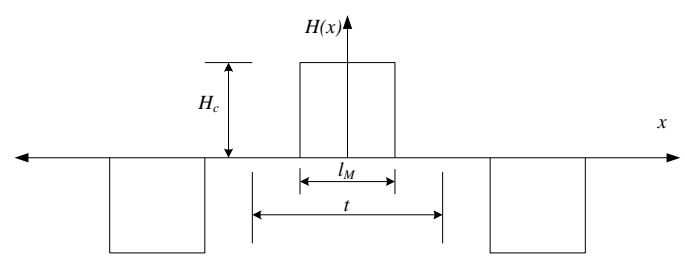

b) Permanent magnet MMF distribution

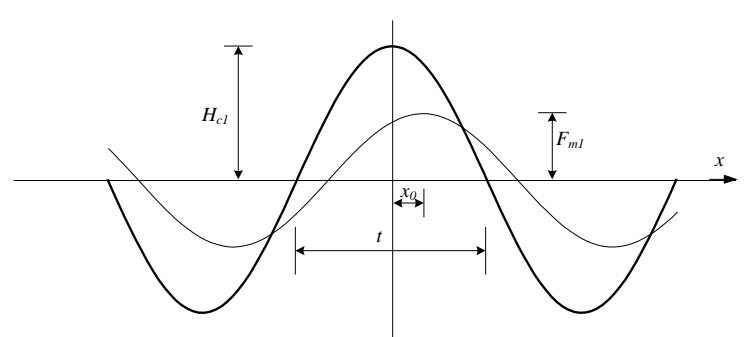

c) Fundamental components of MMF produced by PMs and stator current Fig. 3: PM excitation and MMF distribution

where,

$$
H_{c n}=\frac{4}{\pi} H_{c} \frac{1}{n} \sin \left(n \frac{l_{M}}{\tau} \frac{\pi}{2}\right)
$$

Each coil is supplied by current to produce stator MMF which its fundamental component represented as a function of displacement $x$ as:

$$
(\mathrm{MMF})_{1}=F_{1 m} \cos \left(\frac{\pi}{\tau}\left(x-x_{0}\right)\right)
$$

where,

$$
F_{1 m}=k_{f} I_{m}
$$

$I_{m}$ represents the maximum value of coil current and $k_{f}$ is a constant can be determined by FEM calculations. $x_{0}$ is the mechanical load angle. It is the distance between peak of fundamental component of permanent magnet MMF and peak of fundamental component of MMF produced by coils currents.

The currents of the five coils is a function of displacement $x$ and load angle $x_{0}$ are given by

$$
i_{1}=I_{m} \sin \left(\frac{\pi}{\tau}\left(x-x_{0}+\tau\right)\right)
$$

$$
\begin{aligned}
& i_{2}=I_{m} \sin \left(\frac{\pi}{\tau}\left(x-x_{0}+\frac{\tau}{2}\right)\right) \\
& i_{3}=I_{m} \sin \left(\frac{\pi}{\tau}\left(x-x_{0}\right)\right) \\
& i_{4}=I_{m} \sin \left(\frac{\pi}{\tau}\left(x-x_{0}-\frac{\tau}{2}\right)\right) \\
& i_{5}=I_{m} \sin \left(\frac{\pi}{\tau}\left(x-x_{0}-\tau\right)\right)
\end{aligned}
$$

Depending on Finite-Element Method the thrust force $F_{d}$ can be expressed as in the following equation:

$$
F_{d}=k_{F} \sin \frac{\pi}{\tau} x_{\mathrm{o}}
$$

where, $k_{F}$ can be determined by FEM and it depends on motor dimensions and the fundamental components of MMF for each of PM and coils.

The demand force provides the value of $x_{0}$, so at any position the value of currents of five coils can be determined according to equations from (5) to (9).

\section{4- Dynamic Model and control}

The first step to obtain the dynamic model of the THA is the analysis of blood circulation dynamics. One of the most important equivalent circuits of blood circulation system is Windkessel equivalent circuit [8]. Windkessel models are usually used to describe basic properties of vascular system and to study relationships among hemodynamics variables of blood circulation.

Fig. 4.a is the equivalent circuit of aortic circulation and Fig. 4.b of pulmonary circulation The components of Windkessel model is as follows: $P(t)$ is the applied pressure on aorta or pulmonary artery $\left(\mathrm{N} . \mathrm{m}^{-2}\right.$ or Pa). From Fig. 4.a and 4.b the shift between compression period in aorta and pulmonary can be observed. The period of pressure is obtain from SA node pulses controlled by central nervous system. $Q_{s}(t)$ is the blood flow rate from heart $\left(\mathrm{m}^{3} \cdot \mathrm{sec}^{-1}\right) . Q_{c}(t)$ is the blood flow rate due to compliance $\left(\mathrm{m}^{3} \cdot \mathrm{sec}^{-1}\right), Q_{r}(t)$ is the blood flow rate in peripheral resistance $\left(\mathrm{m}^{3} \cdot \mathrm{sec}^{-1}\right), D$ is to prevent reverse flow of blood. $R_{1}$ is the resistance of major arteries

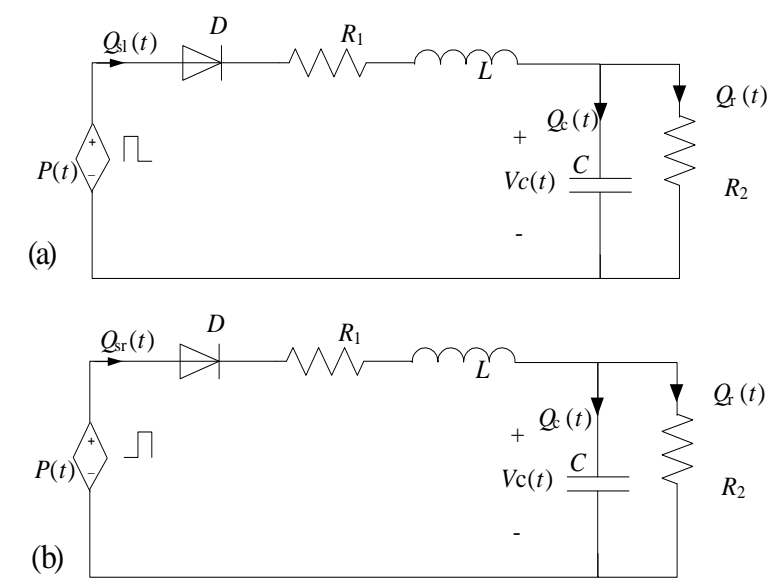

Fig.4: Windkessel model of blood circulation 
(Pa.sec. $\left.\mathrm{m}^{-3}\right) . \quad L$ is the inertia of blood (inertance) $\left(\mathrm{Pa} \cdot \mathrm{m}^{-3} \cdot \mathrm{sec}^{2}\right) . C$ is compliance of veins or arteries $\left(\mathrm{Pa} \cdot \mathrm{m}^{-3}\right)$, $R_{2}$ is peripheral resistance $\left(\mathrm{Pa} . \mathrm{sec} . \mathrm{m}^{-3}\right)$ and $V_{c}$ is the pressure in veins or arteries $(\mathrm{Pa})$. The differential equations that describe the system is as following:

$$
\begin{aligned}
& \frac{d Q_{r}(t)}{d t}=\frac{1}{C R_{2}} Q_{c}(t) \\
& P(t)=L \frac{d Q_{s}(t)}{d t}+R_{1} Q_{s}(t)+R_{2} Q_{r}(t)
\end{aligned}
$$

According to the pusher plate area $A\left(\mathrm{~m}^{2}\right)$ of the total artificial heart the required force $F_{l}(t)$ in Newton is related to applied pressure $P(t)$ as in the following equation:

$$
F_{L}(t)=A P(t)
$$

The linear motor developed force can be determined by the following equation:

$$
F_{d}(t)=F_{L}(t)+m \frac{d^{2} x}{d t^{2}}+B \frac{d x}{d t}
$$

where, $m$ is the mass of motor movable part $(\mathrm{kg})$ and $B$ is the friction(N.sec/m).

Blood flow rate $Q s(t)$ can be related to velocity by the following equation:

$$
\frac{d x}{d t}=\frac{Q_{s}(t)}{A}
$$

From equations (12), (13), (14), (15) the following equation can be obtained:

$$
\frac{d Q_{s}(t)}{d t}=\frac{1}{\left(A L+\frac{m}{A}\right)}\left(F_{d}(t)-\left(\left(A R_{1}\right)+\frac{B}{A}\right) Q_{s}(t)-\left(A R_{2}\right) Q_{r}(t)\right)
$$

The total artificial heart represented in this paper has cardiac stoke volume of $75 \mathrm{ml}$, so with stroke length of 18 $\mathrm{mm}$ the pusher plate area $A$ equal to $0.004167 \mathrm{~m}^{2}$. The end of each pumping phase happens after motor operates in each direction with $18 \mathrm{~mm}$.

The cardiac output equal $75 \mathrm{ml}$ in each pulse. The blood flow rate is a sine wave. So, the Flow rate of blood in each of aorta and pulmonary artery is determined according to the following function:

$$
Q^{*}(t)=\frac{75 \times 10^{-6} \times \pi}{2 t_{s}} \sin \left(\frac{\pi t}{t_{s}}\right)
$$

where, $t_{s}$ is the period of each blood circulation phase i.e. the period of blood compression to aorta which equal the period of blood compression to pulmonary artery. For normal heart rate of $70 \mathrm{bpm}$, the value of $t_{s}$ equals 0.42857 sec. Cardiac output $Q^{*}(t)$ is the reference signal of TAH control system.

Therefore, estimation of the demand forces $F^{*}$ necessary to follow the demand motion pattern is a base for the optimized control parameters control system. According to the PID control law, the demand forces on aorta and pulmonary artery are obtained from the following formula:

$$
F^{*}=k_{p}\left(\ddot{Q}^{*}(t)-\ddot{Q}(t)\right)+k_{i} \int\left(\ddot{Q}^{*}(t)-\ddot{Q}(t)\right) d t+k_{d} \frac{d\left(\ddot{Q}^{*}(t)-\ddot{Q}(t)\right)}{d t}+F_{0}
$$

with

$$
F_{\mathrm{o}}=k_{v c} V_{c}+k
$$

where, $k_{p}$ is the proportional gain, $k_{i}$ is the integral gain, $k_{d}$ is the derivative gain and $F_{0}$ is the force required for the TAH to move according to the demand pattern. The value of $F_{0}$ is proportional to $V_{c}$ the pressure in blood circulation, where the constant of proportionality is $k_{v c}$, additional to a constant value $k$ to enable TAH to move according to the demand pattern. $V_{c}$ represents one of the state variables of the system, where it is observable and measureable [9].

The adjustment of the PID parameters should be performed to obtain appropriate stable and robust control system. Due to the difference of the parameters in each of two blood circulation, the values of controller gains are different although the same pattern of blood flow in the two phases is the same. So, there are six constants should be adjusted.

Fig. 5 illustrates the block diagram of the TAH using the HALSM. The number of heart-beat is determined by the SA node. So, the period of $Q^{*}$ is obtained by multiplying in again $K_{1}$. The PID controller provides the force command and by decoupled law, load angle $x_{0}$ can be determined according equation (10). As mentioned earlier, the five coils current and the mechanical load

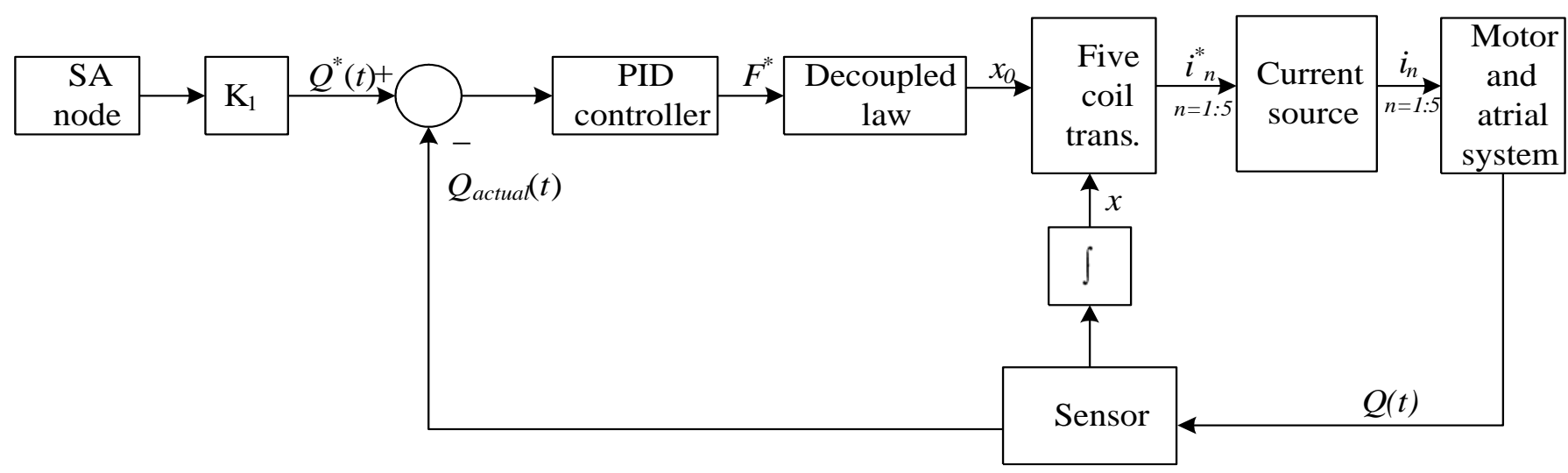

Fig. 5: The block diagram of the TAH using the HALSM 
angle are the control variables of the Halbach array linear motor forces.

According to the decoupled control law, the command five coils current $i_{\mathrm{n}}{ }^{*}$ and command mechanical load angle $x_{0}{ }^{*}$ are obtained. Such values are used to determine the command five coils instantaneous current $i^{*}$, which controls the current source. Hence, the instantaneous five currents are applied to the fixed coils. The traveling wave generated by the five coils current enforces the actuator PM to its new position. The actuator should follows the demand pattern during the full heart-beat, taking into consideration the difference of each of pulmonary circulation parameters and systemic circulation parameter. So, the controller gains should have different values in each phase of motion.

\section{5- Genetic Algorithm to obtain the optimized control parameter}

The GA is applied here to find out the best combination of controller gains to minimize the integrated square error (ISE) between the demand pattern and the produced pattern [10]. The ISE is given by:

$$
\operatorname{ISE}(e)=\int_{0}^{\infty}(e(t))^{2} d t
$$

where

$$
e(t)=Q^{*}(t)-Q(t)
$$

The fitness is taken as the inverse of ISE and the problem becomes the maximization of fitness, given by:

$$
\text { Fitness }=\frac{1}{\operatorname{ISE}(e)}
$$

Through the optimization process, a generation of 20 genes is used. Each gene represents a possible combination of the six gains. Each gene is represented by a binary string of 120 bits. For every gene, the produced force of the TAH actuator is applied on the system for ten cycles of heart operation. Dynamic simulation is performed to find out the $I S E$ for each gene during the ten

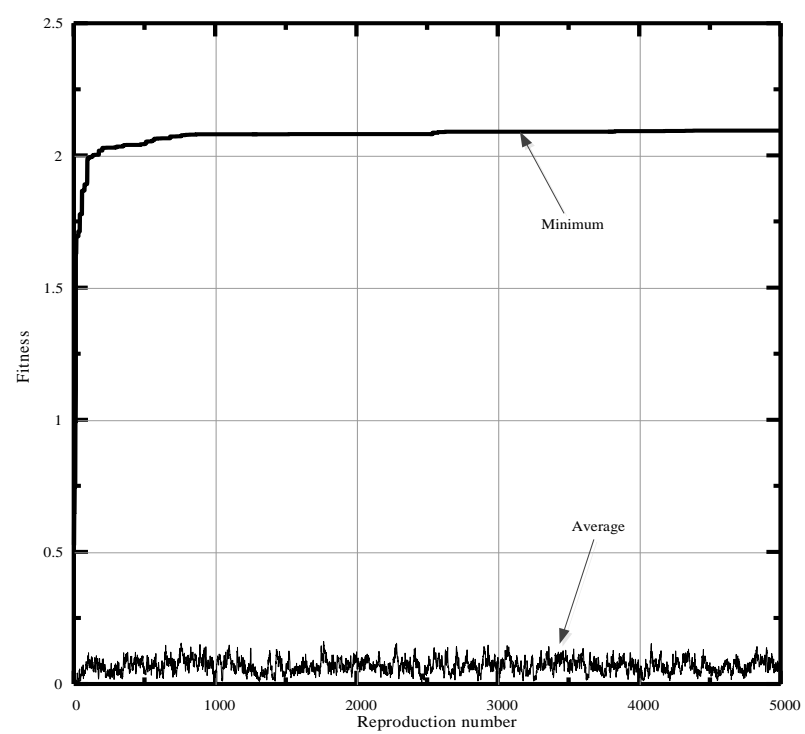

Fig. 6: Minimum and average fitness variation cycles. The programs are developed using $\mathrm{C}++$ programming language and MFC library.

The search process is terminated when the minimum $I S E$ is approximately stable. Fig. 6 shows the minimum and average fitness variation with the generation number.

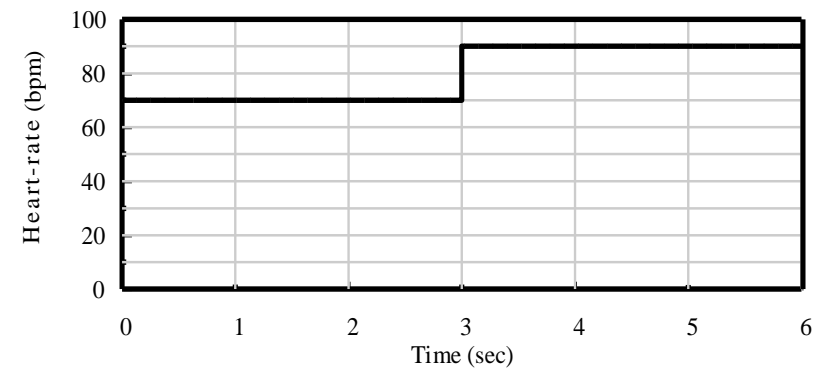

(a) Heart rate

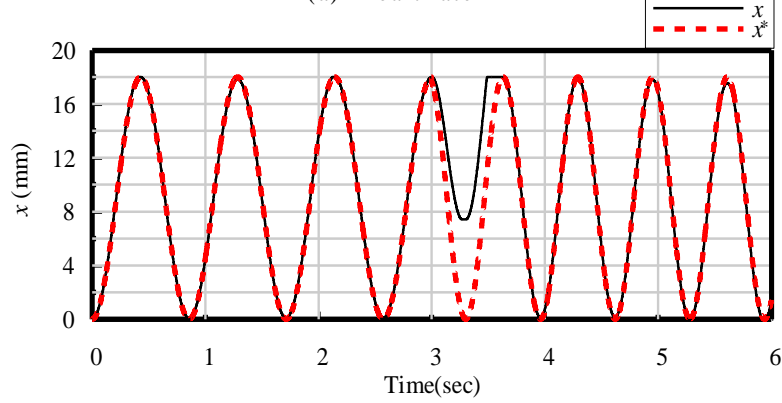

(b) Displacement

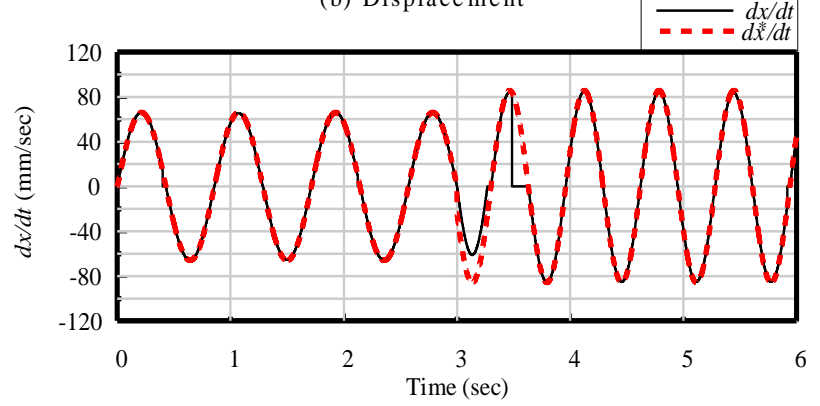

(c) Velocity

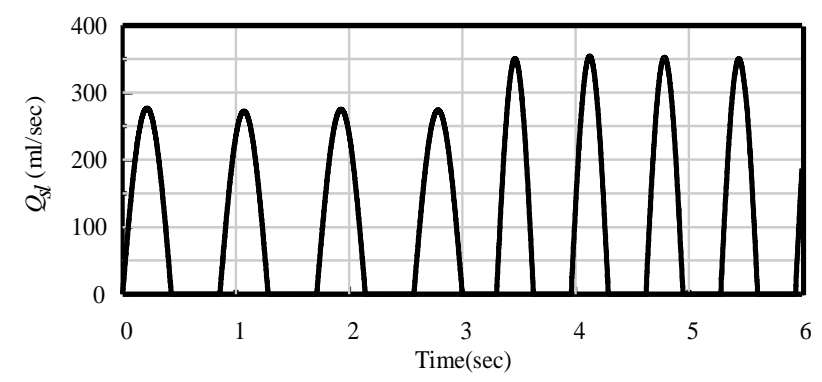

(d) Aortic flow rate

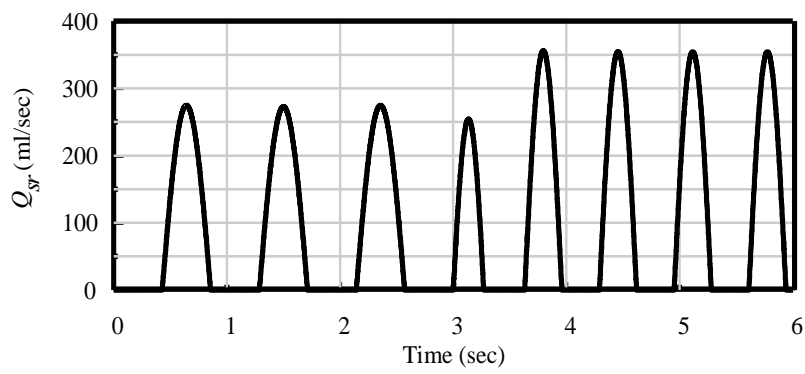

(e) Pulmonary flow rate

Fig. 7: Dynamics of the TAH under optimal control 


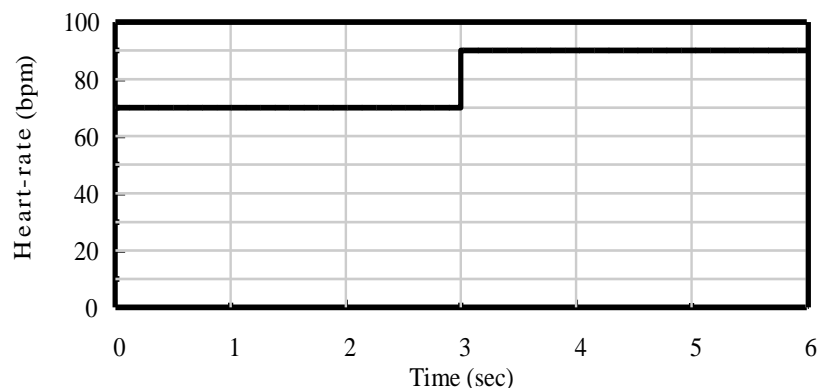

(a) Heart rate

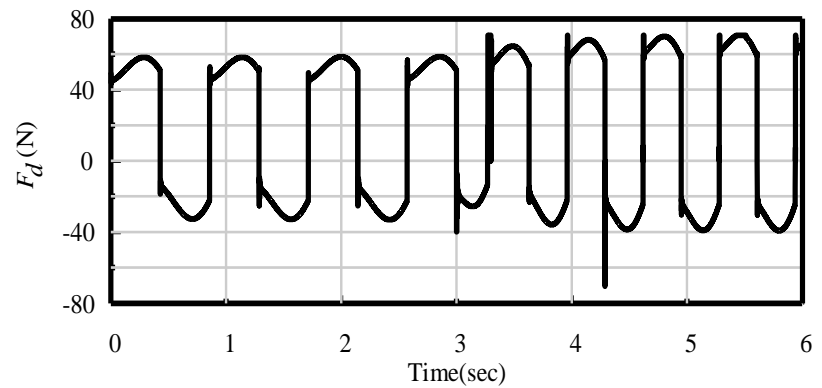

(b) Force

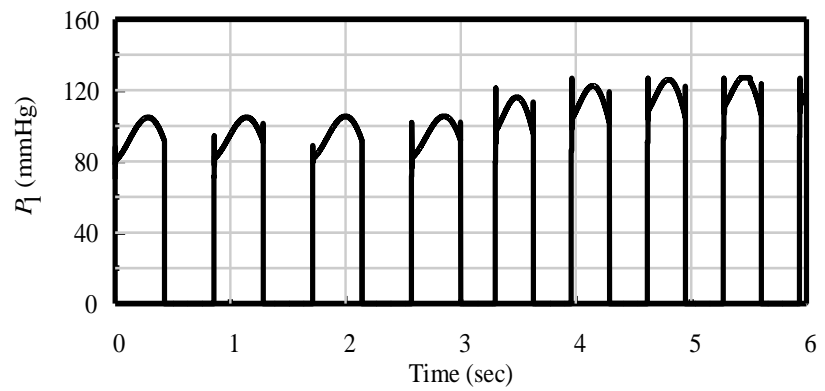

(c) Applied pressure on aorta

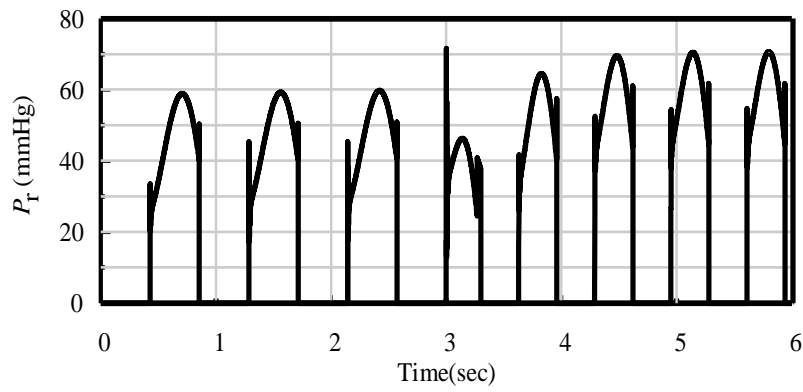

(d) Applied pressure on pulmonary artery

Fig.8: Force and pressure acting on TAH system under optimal control

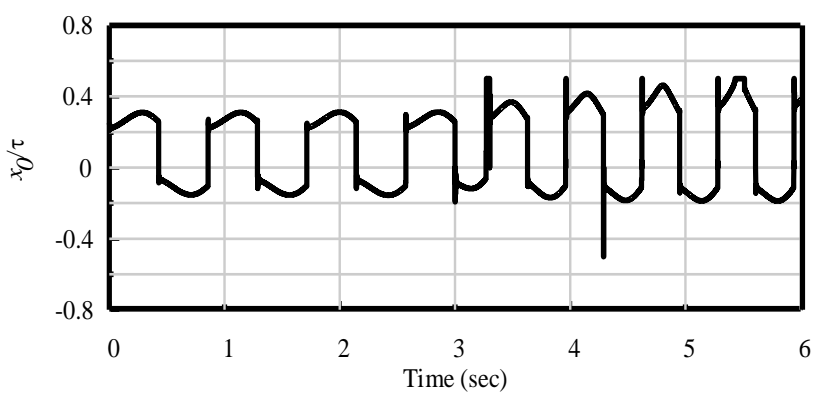

Fig. 9: Mechanical load angle variation under optimal control

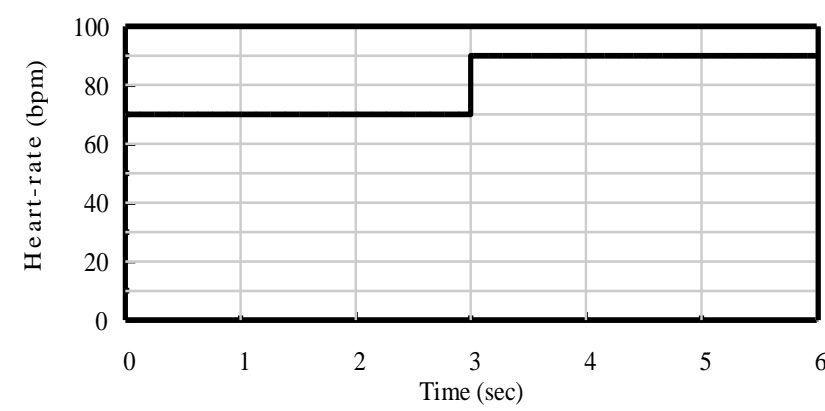

(a) Heart rate

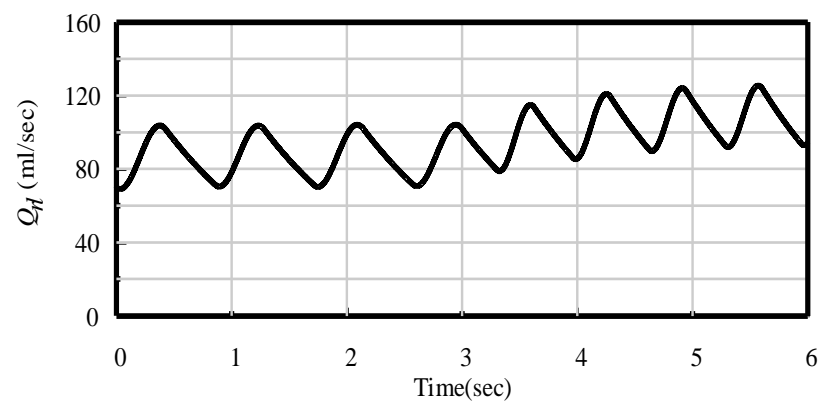

(a) Peripheral arteries flow rate

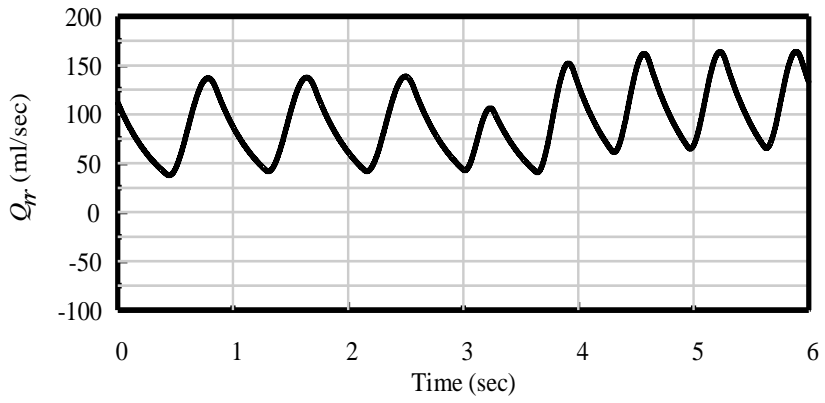

(b) Pulmonary veins flow rate

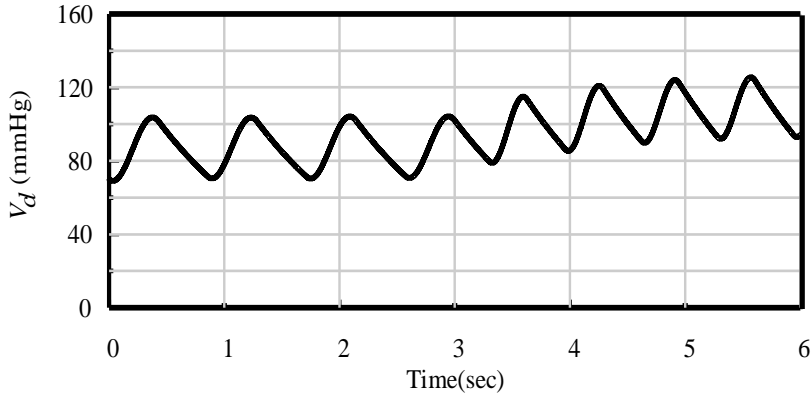

(c) Pressure in arteries

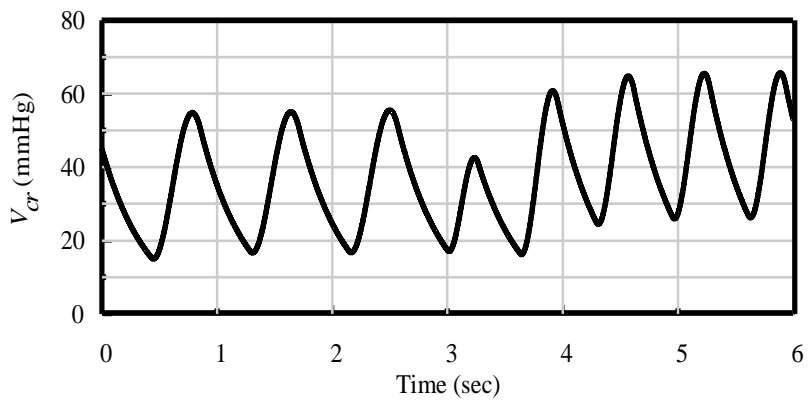

(d) Pressure in veins

Fig. 10: Blood flow and pressure in arteries and veins under optimal control 
After 5000-reproduction process, the maximum fitness was 2.09410 after ten heart cycles. The control gains are found to be: for aortic circulation $k_{p}=0.004674$ $\mathrm{N} / \mathrm{m} / \mathrm{sec}^{2}, k_{i}=7.58985 \mathrm{~N} / \mathrm{m} / \mathrm{sec}, k_{d}=0.000659 \mathrm{~N} / \mathrm{m} / \mathrm{sec}^{3}$, $k_{v c}=0.359549 \mathrm{~N} / \mathrm{mmHg}, k=19.48785 \mathrm{~N}$, for pulmonary circulation $k_{p}=0.013602 \mathrm{~N} / \mathrm{m} / \mathrm{sec}^{2}, k_{i}=27.70892$ $\mathrm{N} / \mathrm{m} / \mathrm{sec}, \quad k_{d}=0.000781 \mathrm{~N} / \mathrm{m} / \mathrm{sec}^{3}, \quad k_{v c}=0.337073$ $\mathrm{N} / \mathrm{mmHg}, k=-1.830852 \mathrm{~N}$.

By applying the demand force according to equation (19) with gain constants provided by the genetic algorithm, the pattern of motion can be obtained. Normal heart rate at rest of $70 \mathrm{bpm}$ has two periods the first is the period of aortic blood compression, the second is the period of pulmonary blood compression. Each of two periods has a time of $0.4286 \mathrm{sec}$. As mentioned earlier in equation (17), the demand blood flow can be represented as a sine wave with an amplitude enable to provide average flow of $75 \mathrm{ml} / \mathrm{beat}$. The following figures illustrate the simulation results of the system under optimal control. Also the required coil currents for the TAH Halbach array actuator will de illustrated. The results are obtained at $70 \mathrm{bpm}$ followed by $90 \mathrm{bpm}$ as a sudden change at the third second as shown in Fig. 7(a) and Fig. 8(a). Fig. 7(b) shows the demand pattern of position represented by the dashed line; the solid line represents the actual position of the actuator with time. The closeness of demand position and actual position can be observed. Fig. 7(c) illustrates the demand pattern of speed which is represented by the dashed line; the solid line represents the actuator actual speed with time. It is notable that there is a good matching between the demand and simulated values of motion. If the heart rate increased suddenly, there is a transient period till the motor follows the demand pattern. There's a separation of actual speed pattern into two phase of motion, the positive represents the flow rate of blood in aorta $Q_{s l}$, the negative speed provide flow rate of blood in pulmonary $Q_{s}$. Fig. 7(d) show the actual flow rate of blood in aorta. Fig. 7(e) show the actual flow rate of blood in pulmonary artery. Fig. 8(b) shows the developed force from the TAH actuator which enables to trace the demand pattern. As shown in Fig. 8(b), there are two levels of force where the large (positive) level for aortic compression and the low (negative) level for pulmonary compression. Fig. 8 (c) illustrates the value of applied pressure on aorta in $\mathrm{mmHg}$ and Fig. 8 (d) illustrates the value of applied pressure on pulmonary artery. The levels of forces and pressure increase because the heart rate increases. The values of coils' currents depend on the value of the mechanical load angle $x_{o}$ according to equation (5) to equation (10). The value of the mechanical load angle depends on the developed force according to equation (10). Fig. 9 shows the variation of mechanical load angle $x_{0}$ as a ratio of pole pitch $\tau$ with time. Fig. 10(a) shows the flow of blood in peripheral arteries Fig. 10 (b) shows the flow of deoxygenized blood in pulmonary veins. Fig. 10 (c) illustrates the pressure in peripheral arteries $V_{c l}$. Fig. 10 (d) shows the pressure in lung veins. The obtained results correspond with the natural blood circulation of a human adult [6].

According the value of load angle, the value of coils excitation can be determined according to equations (5) to (9). Fig. 11 illustrates the coils excitation. The currents

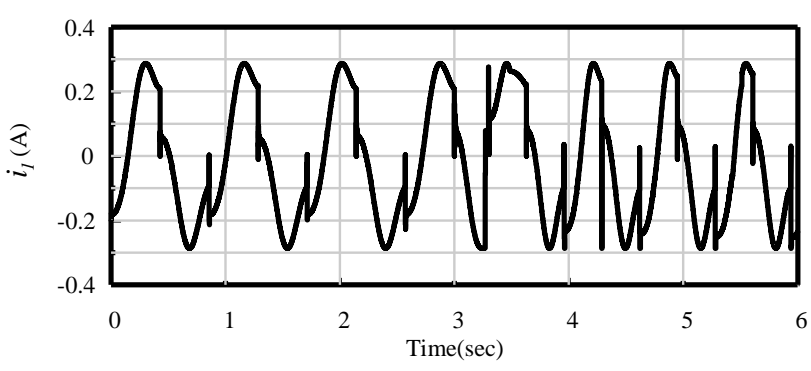

(a) Current of coil 1

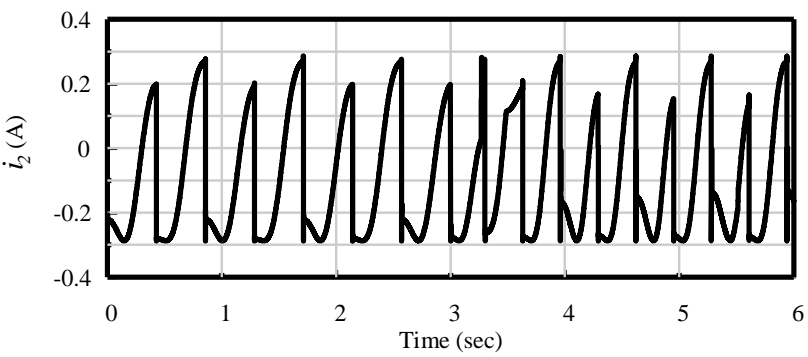

(b) Current of coil 2

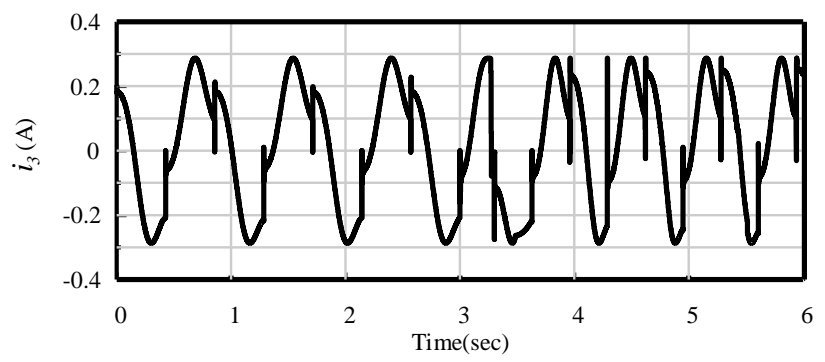

(c) Current of coil 3

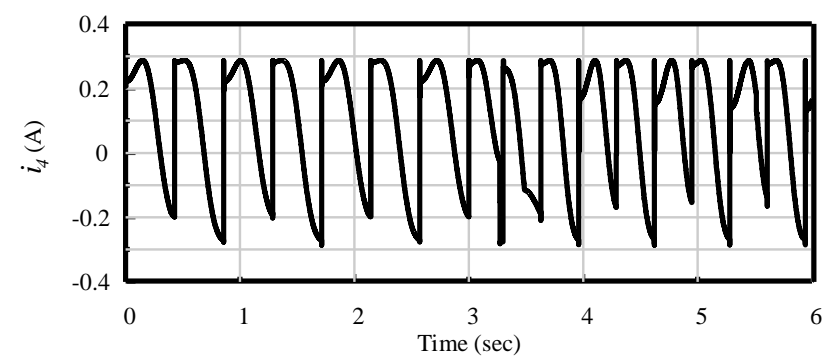

(d) Current of coil 4

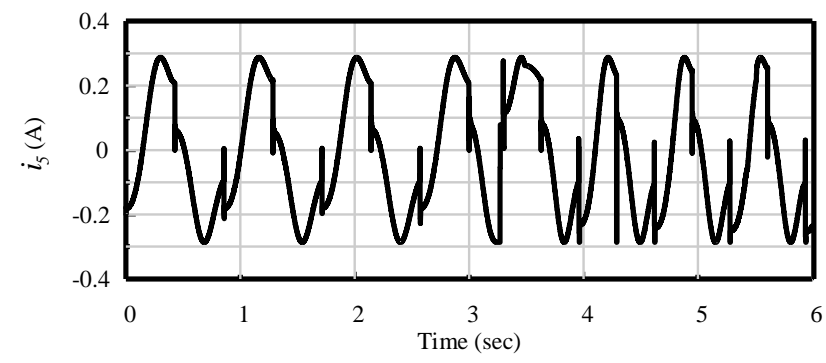

(e) Current of coil 5

Fig. 11: Coils excitation required to develop the optimal performance 
frequency increases due to the increasing of the heart rate. These currents represent the reference value that the current source should follow.

\section{6- Conclusion}

A proper design of HALSM for the TAH is established. This design has a proper volume suitable to be implanted in the human body. This design produces high level of forces that it can drive the human blood circulation with minimum amount of losses.

The analytical model of HALSM developed force is presented using the space harmonic analysis of multilayer boundary condition. The FEM provides the parameters of the proven formula. According to this analysis, the currents to obtain the required force can be determined.

The dynamic model of combined HALSM and blood circulation is achieved taking into account the hemodynamic parameters and the HALSM parameters. Therefore, the consistent PID controlled is developed to enable the TAH system of developing certain blood flow rate.

The GA is used to obtain the optimized control parameter with a proper performance index. The GA provides the PID parameters at maximum fitness value. The performance of TAH driven by HALSM is developed at normal heart-rate and under sudden change. The results improves the effectiveness of the optimal control to enable the TAH of providing the reference motion pattern.

The dynamics of the TAH using HALSM is obtained under optimal control. The developed forces of the TAH and the blood pressures are obtained. Under sudden change of heart-rate, the values of forces and pressure are increased. The developed force doesn't be increase above $70.5 \mathrm{~N}$ due to design limitations. So for heart-rate exceeds $90 \mathrm{bpm}$, the performance of the TAH doesn't fulfill the required flow rate.

The current patterns of each coil have been obtained. These currents enable to develop the required force. The high change in current demand requires a fast acting power converter to keep the coils current value matched with the controller demand value. Such converter should have the following features: (1) relatively, high dc bus voltage to allow for fast response (2) sufficient current and power demand. The frequency of the required currents is increased by the heart rate increasing.
The future work will focus on the performance of the TAH driven by HALSM under high sudden change and on the design optimization of such actuator.

\section{7- Reference}

[1] Kristin Friedline and Pamela Hassinger "Total Artificial Heart Freedom Driver in a Patient With End-Stage Biventricular Heart Failure" AANA Journal, April 2012, Vol. 80, No. 2

[2] S Thomas Finocchiaro, Thomas Butschen_, Paul Kwant, Ulrich Steinseifer, Thomas Schmitz-Rode, Kay Hameyer, and Marc Leßmann "New Linear Motor Concepts for Artificial Hearts "IEEE TRANSACTIONS ON MAGNETICS, VOL. 44, NO. 6, JUNE 2008.

[3] Makoto Aakamora, et al: "Transient response of remnant atrial heart rate to step changes in artificial heart output". The Japanese Society for Artificial Organs 2002. J Artif Organs (2002) 5:6-12

[4] A. E. ElGebaly, M. El-Nemr and M. ElKhazendar "Halbach array linear motor actuator for the total artificial heart" 13th International Middle East Power System Conference. MEPCON,2009

[5] K. Halbach, "Design of permanent magnet multipole magnets with oriented rare earth cobalt material," Nucl. Instrum. Meth., vol. 169, pp. 1-10, 1980.

[6] John K-J Li "DYNAMICS of the VASCULAR SYSTEM" Series on Bioengineering \& Biomedical Engineering - Vol. I, World Scientific Publishing Co. Re. Ltd, 2004

[7] K. Yoshida, and Weh: "Theory of Controlled PM Linear Synchronous Motor", Proc. Of $10^{\text {th }}$ inter. Conf. on Maglev'88, pp. 259-268, 1988.

[8] Nikos Stergiopulos, Berend E. Westerhof, and Nico Westerhof "Total arterial inertance as the fourth element of the windkessel model" Am J Physiol Heart Circ Physiol 276: H81-H88, 1999; Vol. 276, Issue 1, H81-H88, January 1999

[9] Ivort T. Gabe, et al: "Measurement of Instantaneous Blood Flow Velocity and Pressure in Conscious Man with a Catheter-Tip Velocity Probe" Journal of American heart association, Circulation 1969;40;603-614

[10] D. Goldberg: 'Genetic Algorithms in Search, optimization and machine learning", AddisonWesley 1989. 